\title{
La fabrique du « soldat-citoyen » à travers la conscription en Turquie
}

The Making of the "citizen-soldier" through conscription in Turkey

\section{Sümbül Kaya}

\section{(2) OpenEdition \\ 1 Journals}

Édition électronique

URL : http://journals.openedition.org/ejts/2922

DOI : $10.4000 /$ ejts.2922

ISSN : $1773-0546$

\section{Éditeur}

EJTS

\section{Référence électronique}

Sümbül Kaya, « La fabrique du « soldat-citoyen » à travers la conscription en Turquie », European Journal of Turkish Studies [En ligne], 8 | 2008, mis en ligne le 04 novembre 2009, consulté le 16 février 2020. URL : http://journals.openedition.org/ejts/2922 ; DOI : 10.4000/ejts.2922 


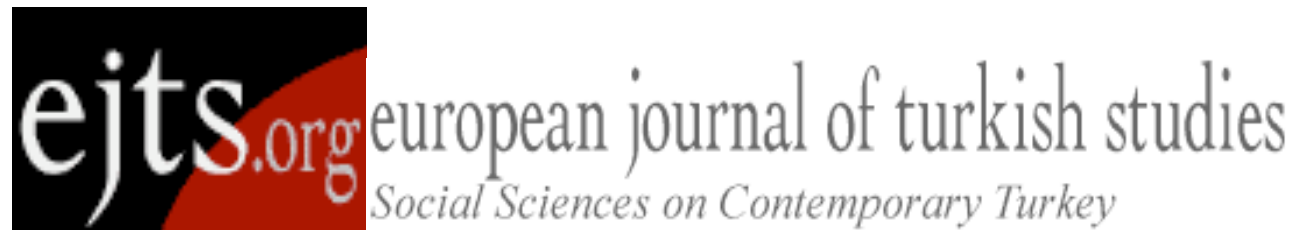

Kaya, Sümbül (2009) 'La fabrique du 'soldat-citoyen' à travers la conscription en Turquie', European Journal of Turkish Studies, Thematic Issue N 8 , No. 8 | Surveiller, normaliser, réprimer, URL : http://www.ejts.org/document2922.html To quote a passage, use paragraph (§).

\title{
La fabrique du 'soldat-citoyen' à travers la conscription en Turquie Sümbül Kaya
}

\begin{abstract}
This paper examines how the disciplinary mechanisms of the Turkish army can produce a transformation in the habitus of the conscripts. As a total institution, the military institution integrates and normalizes the conscripts through rites, control of time, horizontal surveillance between conscripts, and corporal punishments. The paper argues that the effects of military service are, nonetheless, not homogenous. This is primarily because of differences in the initial social profile of the conscripts, which produce different resources. Thus it is possible to suggest a typology of the different adaptative strategies of the conscripts. Finally, the article argues that this socialization in the military is part of a larger process of the building of a national habitus in Turkey.
\end{abstract}


Kaya, Sümbül (2009) 'La fabrique du 'soldat-citoyen' à travers la conscription en Turquie', European Journal of Turkish Studies, Thematic Issue $\mathrm{N}^{\circ} 8$, No. 8 | Surveiller, normaliser, réprimer, URL : http://www.ejts.org/document2922.html To quote a passage, use paragraph (§).

Bernard Lahire, critique la notion d'habitus1 de Pierre Bourdieu en introduisant la notion d"acteur pluriel'. En effet, pour cet auteur, les acteurs sont 'le produit de l'expérience - souvent précoce - de socialisation dans des contextes sociaux multiples et hétérogènes' (Lahire 2001 : 60), ils sont donc dotés d'une pluralité dispositionnelle ou d'un patrimoine de dispositions hétérogènes plutôt que d'un habitus homogène (Lahire 2003). Cependant, Bernard Lahire envisage deux situations exceptionnelles où les dispositions des individus peuvent être davantage homogénéisées : les institutions totales et les sociétés traditionnelles. Le concept bourdieusien d'habitus ne serait ainsi opératoire que dans ces contextes particuliers. C'est pourquoi l'institution militaire nous paraît être un terrain intéressant pour confronter ces différentes théories. Les dispositifs disciplinaires sont inhérents à une institution totale2 et, comme le souligne Bernard Lahire, il s'agit d'une institution 'encastrée dans une société à forte différenciation'. Dès lors, les dispositions sociales acquises au sein des institutions totales peuvent être durables et transposables à d'autres secteurs de la société (Lahire 2001 : 39). Pourrait-on alors penser que les mécanismes disciplinaires d'une institution peuvent produire des habitus tant nationaux que sociaux3 là où il y avait pluralité dispositionnelle ? En fondant notre analyse sur le cas turc, notre hypothèse est que les procédures disciplinaires mises en place dans les institutions totales comme l'armée4 peuvent produire un ethos

1 Pierre Bourdieu définit l'habitus comme 'un système de dispositions ouvert qui va être constamment soumis à des expériences, et du même coup transformé par les expériences' (Bourdieu et al 1989: 57-58). II considère par ailleurs que 'les conditionnements associés à une classe particulière de condition d'existence produisent des habitus, systèmes de dispositions durables et transposables, structures structurées prédisposées à fonctionner comme des structures structurantes, c'est-à-dire en tant que principes générateurs et organisateurs de pratiques et de représentations...' (Bourdieu $1980: 88$ ).

${ }^{2}$ La définition donnée par Goffman de l'institution totale est la suivante : 'un lieu de résidence et de travail où un grand nombre d'individus, placés dans la même situation, coupés du monde extérieur pour une période relativement longue, mènent ensemble une vie recluse dont les modalités sont explicitement et minutieusement réglées' (Goffman 1968 : 1).

${ }^{3}$ Nobert Elias définit l'habitus national comme 'les caractéristiques de l'identité collective nationale " et l'habitus social comme 'la structure de la personnalité de l'individu'. 'Les caractéristiques de l'identité collective nationale - ce que nous appelons le 'caractère national' - est une strate de l'habitus social très profondément et très solidement ancrée dans la structure de la personnalité de l'individu .... L'enracinement profond de différents caractères nationaux et la conscience de sa propre identité collective nationale, très étroitement liée à ce caractère, pourraient être un exemple assez clair de la façon dont l'habitus social de l'individu fournit le terrain sur lequel se développent des différences individuelles purement personnelles' (Elias 1991 : 273).

${ }^{4}$ Levent Ünsaldı rapproche également l'organisation de l'armée turque de 'l'institution totale' même s'il souligne que cette appartenance est volontaire pour les militaires de carrière (Ünsaldı 2005 : 
Kaya, Sümbül (2009) 'La fabrique du 'soldat-citoyen' à travers la conscription en Turquie', European Journal of Turkish Studies, Thematic Issue $\mathrm{N}^{\circ} 8$, No. 8 | Surveiller, normaliser, réprimer, URL : http://www.ejts.org/document2922.html To quote a passage, use paragraph (§).

militaire permettant la réactivation d'un habitus national conforme à l'idéologie kémaliste. L'armée turque est en effet une armée de conscription5 et elle joue un rôle central dans le système politique, à la fois comme acteur du jeu institutionnel (y compris par des coups d'Etat) et comme lieu de formation du sentiment national. Toutefois, les dynamiques internes de l'institution, les dotations initiales en capitaux des individus, mais surtout les résistances et adaptations secondaires des appelés interdisent la production d'un ethos militaire uniformément docile et donc la production d'un habitus national uniforme. Nous chercherons donc à mettre en évidence les effets souhaités de ce processus de socialisation secondaire 6 et nous dégagerons quelques pistes d'analyses sur les effets différenciés de la socialisation dans les institutions totales en fonction des capitaux initiaux des individus.

[2] Cette recherche s'appuie sur une centaine d'entretiens semi-directifs ou ouverts (récits de vie centrés sur l'expérience militaire) réalisés entre 2005 et 2007 dans la province anatolienne de Kayseri. Tous les enquêtés sont nés dans ce département et ils y vivaient au moment des entretiens. La religion joue un rôle important à Kayseri dans la mesure où elle structure largement la vie sociale et politique des habitants7. Nous avons constitué un échantillon composé de différentes variables

189). En ce qui concerne les conscrits, le caractère volontaire n'est plus valable puisqu'il s'agit d'une obligation. Ainsi conformément à la définition donnée par Goffman les appelés sont bien 'coupés du monde extérieur pour une période relativement longue, mènent ensemble une vie de recluse dont les modalités sont explicitement et minutieusement réglées'.

${ }^{5}$ La durée de base du service est actuellement de 15 mois pour tous ceux qui ont un niveau universitaire inférieur à 4 ans. Pour les personnes qui possèdent un niveau universitaire de quatre ans et plus, la durée du service sera de 12 mois (officier de réserve) ou 6 mois (hommes du rang) en fonction des besoins de l'armée. Pour ceux qui vivent à l'étranger, le service sera d'un mois et une somme d'argent devra être payée à l'institution militaire. La durée de ce service national - autrefois de 24, puis de 18 mois et aujourd'hui de 15 mois pour les hommes du rang - fait que cette expérience a des effets profonds sur les appelés, ce qui nous conduit à interroger sur ce moment clé de la socialisation des hommes turcs.

${ }^{6} \|$ nous paraît donc intéressant de rapprocher le service national du concept de 'socialisation secondaire' construit par P. Berger et T. Luckmann. Les auteurs définissent la socialisation secondaire comme 'l'intériorisation de 'sous-mondes' institutionnels ou basés sur des institutions. Son étendue et ses caractéristiques sont dès lors déterminées par la complexité de la division du travail et de la distribution sociale de la connaissance qui lui est rattachée' (Berger et al 1996 : 177 257).

${ }^{7}$ Le Parti de la Justice et du Développement (Adalet ve Kalkınma Partisi) a obtenu $70 \%$ des voix lors des élections municipales du 28 mars 2004 et $65,73 \%$ des voix lors de l'élection présidentielle du 28 août 2007. Le Parti du mouvement national 'Milliyetçi Hareket Partisi' arrive en deuxième position en obtenant $17 \%$ lors des élections municipales de 2004 et $16 \%$ des voix à l'élection présidentielle de 
Kaya, Sümbül (2009) 'La fabrique du 'soldat-citoyen' à travers la conscription en Turquie', European Journal of Turkish Studies, Thematic Issue $\mathrm{N}^{\circ} 8$, No. 8 | Surveiller, normaliser, réprimer, URL : http://www.ejts.org/document2922.html To quote a passage, use paragraph $(\S)$.

mais nous ne ferons pas une étude exhaustive intégrant l'influence de tous ces facteurs dans ce travail. Notre corpus d'entretiens pourrait se diviser en trois groupes : les hommes qui se préparaient à partir au service militaire, ceux qui étaient en train de l'effectuer et ceux qui en sont revenus. Pour certains enquêtés, nous avons pu suivre ces trois étapes successives. Par ailleurs, les entretiens ont concerné trois différentes générations de conscrits, ce qui nous a permis de saisir l'évolution de l'expérience du service militaire dans la durée. II nous a également été possible d'interroger ces trois générations sur leurs expériences du service militaire au sein d'une même famille (grand-père, père, fils). Nous avons élaboré un échantillon où sont représentées les différentes catégories socioprofessionnelles, les différents lieux d'habitation (urbain-rural) et les diverses sensibilités politiques et idéologiques. L'entretien a pu à certains moments être complété par un témoignage de la mère ou de la femme de l'enquêté. II nous a enfin été possible de nous entretenir avec des officiers chargés de la formation des appelés et de faire des observations au sein d'une caserne. Tous ces entretiens ont été effectués en dehors de la caserne et, pour la partie de notre échantillon constituée des appelés qui en sont revenus, les entretiens ont parfois été réalisés plusieurs années après le retour du service militaire. La durabilité des dispositions acquises lors du service militaire reste cependant difficile à évaluer notamment en raison des socialisations contradictoires qui perdurent après le service. Ces dernières pouvant non seulement atténuer, amplifier ou réactiver des dispositions, mais aussi les mettre en sommeil.

[3] Nous verrons d'abord à travers l'analyse des rites de départ au service national et l'étude des représentations collectives concernant l'institution militaire et le service militaire qu'il existe une certaine légitimité sociale à l'encasernement, ce qui nous conduira à considérer le service national à la fois comme un rite de passage' et un 'rite d'institution'. L'étude des rites d'admission nous permettra également de saisir comment l'institution intègre l'appelé et comment elle accomplit une mise en condition au moyen de pratiques uniformisatrices. Nous nous attacherons ensuite à décrire les dispositifs disciplinaires qui visent à faire intérioriser aux appelés un ethos militaire normalisé. Ce dernier nous semble être un outil indispensable afin de réactiver chez les appelés les composantes de l'habitus national conforme à l'idéologie kémaliste : au-delà de l'apprentissage des

2007. URL : http://www.yerelsecim.com. Au niveau local, les cadres politiques du Parti se disent êtres conservateurs sur la question des mœurs et libéraux sur plan économique. Les résultats du parti du mouvement nationaliste à Kayseri nous montrent qu'une partie des Kayseriotes adhèrent aux idées d'extrême droite qui prônent une certaine conception de la nation turque au niveau ethnique, linguistique et religieuse. Sur la place de la religion dans cette province (Kaya 2004). 
Kaya, Sümbül (2009) 'La fabrique du 'soldat-citoyen' à travers la conscription en Turquie', European Journal of Turkish Studies, Thematic Issue $\mathrm{N}^{\circ} 8$, No. 8 | Surveiller, normaliser, réprimer, URL : http://www.ejts.org/document2922.html To quote a passage, use paragraph (§).

techniques militaires, l'éducation et la formation auxquelles sont soumis les appelés véhiculent une certaine conception de la nation en Turquie. Nous analyserons également les effets souhaités de cette éducation et mettrons ainsi en évidence les objectifs officiels de l'institution militaire. Enfin, nous montrerons qu'en dépit de ces dispositifs, les expériences des appelés restent très différentes - tant en raison de leurs affectations que de leur rapport à l'institution - démontrant le caractère hétérogène de cette force socialisatrice sur des individus aux origines et aux trajectoires multiples.

\section{I 'partir c'est mourir un peu' : les rites de départ et d'intégration comme symboles du début d'une nouvelle vie}

[4] L'armée et le service militaire sont des institutions bénéficiant d'une légitimité sociale au sein de la population. Qu'est ce qui prédispose les appelés turcs à accepter l'encasernement? D'abord, nous décrirons les représentations collectives de nos enquêtés à l'égard de ces institutions ce qui nous permettra de considérer le service militaire à la fois comme un 'rite de passage et un 'rite d'institution' Par ailleurs, ces représentations collectives s'accompagnent de certains rites de départ dont l'analyse montrera qu'ils tendent à favoriser l'acceptation de l'idée d'encasernement par les appelés. Enfin, la description des mécanismes d'intégration de l'appelé permettra de comprendre comment se réalise concrètement la 'mise en condition' (Goffman 1968) du nouvel arrivant au sein de la caserne.

[5] Les entretiens effectués montrent qu'il existe au sein de la population turque un sentiment favorable non seulement à l'égard du service militaire mais aussi à l'égard de l'institution elle-même. Le soldat est souvent admiré ; personne n'hésitera à l'aider, à lui octroyer une réduction, à lui offrir un repas ou à le véhiculer gratuitement. II n'est pas rare de voir les parents sortir avec leur fils vêtu de l'uniforme militaire que les familles peuvent acquérir simplement sur les marchés au même titre que les costumes traditionnels. Toute une historiographie mettant en scène l'idée de 'nation-en-armes' ou l'idée de nation militaire, Asker millet, est exaltée au sein de la société turque. Les références actuelles aux batailles et conflits passés comme la guerre des Dardanelles durant la première guerre mondiale et la guerre de libération (1920), renforcent cette idée, illustrée par la célèbre formule de Mustafa Kemal Atatürk 'chaque turc naît soldat'. Comme le souligne Ayşe Gül Altınay, 'La nation turque a été inventée comme une 'nation militaire'. La conscription et l'éducation militaire obligatoires ont favorisé sa création et son renforcement' (Altınay 2004: 161). Ce mythe de la 
Kaya, Sümbül (2009) 'La fabrique du 'soldat-citoyen' à travers la conscription en Turquie', European Journal of Turkish Studies, Thematic Issue $\mathrm{N}^{\circ} 8$, No. 8 | Surveiller, normaliser, réprimer, URL : http://www.ejts.org/document2922.html To quote a passage, use paragraph (§).

nation militaire a pour effet de favoriser l'adhésion des appelés à l'institution, alors même que la conscription renforce les liens entre l'armée et le reste de la société. La vie politique et sociale en Turquie est réellement imprégnée de cette culture militaire.

[6] Dans les représentations collectives, le service militaire est considéré comme une épreuve obligatoire à laquelle les jeunes hommes de la nation ne peuvent échapper. Tous les enquêtés disent accomplir leur devoir patriotique et, à quelques exceptions près, ils ne sont pas favorables à sa suppression.8 Finalement, dans le contexte turc, le service militaire est également une 'obligation collective qui ne se discut[e] guère' (Bessin 2002: 9). Dans les représentations collectives des appelés, le service militaire est bien le lieu de l'apprentissage de leur virilité et c'est à ce moment qu'ils apprennent à devenir des hommes. L'adage populaire 'on ne donne pas de fille ni de travail à celui qui n'a pas fait son service' illustre bien cette idée. Le service militaire est-il alors un 'rite de passage' (Van Gennep 1981) défini comme un passage du statut d'enfant au statut d'homme ou bien est-il un 'rite d'institution' qui instaure 'une différence durable entre ceux que ce rite concerne et ceux qu'il ne concerne pas' (Bourdieu 1982 : 58) ? Nous verrons que le service national en Turquie peut à la fois être considéré comme un 'rite de passage' et comme un 'rite d'institution'. A la fin du service, le conscrit deviendra un homme et perdra ainsi son statut d'enfant et même d'adolescent; il deviendra également homme en se distinguant du monde féminin non soumis à la conscription. Pendant le service militaire, l'apprentissage des valeurs guerrières, la pratique des combats, le maniement des armes, et la camaraderie virile renforcent le sentiment de virilité des appelés. La présence d'une culture militaire dans la vie politique et sociale en Turquie et les représentations collectives sur cette épreuve militaire peuvent faciliter l'acceptation de normes et de règles militaires. L'image du service militaire et de l'armée dans la société incite en effet à penser que le service militaire est à la fois un rite de passage et un rite d'institution dans la société turque.

[7] Le manuel scolaire de second cycle intitulé La science de la société (Yıldırım et al 2004 : 132-133) présente le départ pour le service militaire comme un moment de festivité, ce qui correspond effectivement à ce que nous avons constaté sur notre terrain et recueilli lors de nos entretiens. La célébration du départ au service militaire s'est institutionnalisée et a pris de l'importance surtout depuis ces dix dernières années. II s'agit d'une fête où l'appelé est au centre de

${ }^{8}$ La situation d'entretien peut éventuellement fausser la réponse des enquêtés à cette question. De plus, ce refus de supprimer le service national n'est certainement pas généralisable à tous les hommes de Turquie. 
Kaya, Sümbül (2009) 'La fabrique du 'soldat-citoyen' à travers la conscription en Turquie', European Journal of Turkish Studies, Thematic Issue $\mathrm{N}^{\circ} 8$, No. 8 | Surveiller, normaliser, réprimer, URL : http://www.ejts.org/document2922.html To quote a passage, use paragraph (§).

toutes les attentions, au même titre qu'un anniversaire. A Kayseri, un mois avant le départ, les proches de l'appelé commencent à lui rendre visite, des cadeaux lui sont offerts, de l'argent de poche et des pièces en or lui sont données9. Pour les plus pratiquants, un repas est organisé avant le départ en présence d'un imam qui récite une prière afin de demander la protection de l'appelé. S'il n'y a pas d'imam, la famille et les invités feront tout de même une prière. Dans certains cas, les familles mettent du henné dans la main de l'appelé avant son départ, ce qui signifie qu'il est sacrifié pour la nation10. Le jour du départ, des joueurs de tambours et de clarinette (davul zurna) sont invités et animent les moments d'adieux. L'appelé est accompagné par ses proches jusqu'à la gare routière. La tradition veut que les proches le jettent en l'air en criant la célèbre formule d'Atatürk 'le plus grand soldat est notre soldat! (En büyük asker bizim asker!). Ensuite, l'appelé monte dans le bus, qui est arrêté par les proches chantant l'hymne national. Cependant, les observations pratiquées dans les gares routières montrent que la séparation avec la famille et le départ sont un événement moins festif que d'autres célébrations, même si certains rituels sont pratiqués.

[8] L'intégration au sein de l'armée passe ensuite par l'isolement. En effet, la société militaire est un univers clos qui intègre les civils par la conscription mais les isole du reste de la société en général pendant 15 mois. L'institution délimite l'espace social en imposant une séparation entre le monde civil et le monde militaire pendant la période des classes, et une quasi-séparation après les classes car les permissions de sortie en fin de semaine sont étroitement contrôlées. Les clôtures symbolisent cette séparation et la superficie des zones militaires est très importante à Kayseri où l'université est accolée à la zone militaire. Le mot turc pour signifier 'se rendre à la caserne' ou à son unité est teslim olmak; on peut le traduire comme 'se soumettre' ou 'se rendre' mais il est également utilisé lorsqu'une personne se rend à la police. II y a donc l'idée de soumission à une autorité, en l'occurrence à l'autorité militaire. Des rites d'admission, qui s'apparentent souvent

${ }^{9}$ Ces célébrations n'ont pas lieu lorsque la date de départ au service national est retardée de plusieurs années, car l'idée d'un passage à l'âge adulte ne fait alors plus sens.

10 Dans la tradition turque, l'usage du henné se fait dans trois cas (en dehors d'un usage purement esthétique) :

lorsqu'une femme se marie parce qu'elle est considérée comme un sacrifice offert à son mari, lorsqu'un homme se marie parce qu'il est considéré comme un sacrifice offert à Allah, lorsqu'un homme part au service parce qu'il est considéré comme un sacrifice pour la nation. L'usage du henné renvoie symboliquement à la figure religieuse d'Abraham qui était prêt à sacrifier son fils pour Dieu qui alors remplacera le fils par un bélier. 
Kaya, Sümbül (2009) 'La fabrique du 'soldat-citoyen' à travers la conscription en Turquie', European Journal of Turkish Studies, Thematic Issue $\mathrm{N}^{\circ} 8$, No. 8 | Surveiller, normaliser, réprimer, URL : http://www.ejts.org/document2922.html To quote a passage, use paragraph (§).

à des 'techniques de mortification' (Goffman 1968), sont mis en place par l'institution pour accueillir les nouveaux appelés et participent à leur mise en condition.

[9] En rentrant à la caserne, l'appelé est tout d'abord non seulement dépossédé de son identité mais aussi de ses rôles sociaux (professionnels et familiaux). Afin de ne pas les inquiéter, certains appelés ne disent pas à leurs parents où ils vont être affectés après les classes, surtout quand ils partent combattre la guérilla du PKK dans l'Est. Pendant la durée des classes et jusqu'au jour du serment, l'appelé n'a pas le droit de sortir de l'enceinte de la caserne. Cette période peut varier d'un mois à 40 jours en fonction des différents statuts. Au cours du premier et du deuxième jour, il est conseillé à l'appelé de téléphoner à sa famille afin de la rassurer et l'institution militaire signale par une lettre aux parents qu'ils ne doivent pas informer directement leur fils d'une mauvaise nouvelle mais plutôt en aviser l'institution. En général, après la période des classes, l'appelé a la possibilité de prendre 30 jours de congés, mais certains préfèrent ne pas utiliser cette possibilité afin de terminer leur service au plus tôt.

[10] Une fois que l'appelé est admis à pénétrer dans l'enceinte de la caserne, il participe à ce que Goffman nomme 'les cérémonies d'admission'. II est d'abord fouillé et ses effets personnels sont contrôlés, certains d'entre eux confisqués (comme les livres, les affaires de toilettes, les téléphones portables, les radios...). L'appelé est donc dépouillé de ses biens qui seront remplacés par des 'objets de série, de caractère uniforme et uniformément distribués' (Goffman 1968 : 61). Effectivement, chaque personne reçoit un sac contenant un nécessaire de toilette, des sousvêtements, des serviettes, des coupe-vents, des uniformes d'été et d'hiver ainsi que des chaussettes, un bloc note et un crayon. Ensuite, le matériel devra être rangé suivant un ordre précis dans le placard, qu'il est interdit de fermer à clé.

[11] La rupture avec le monde civil passe aussi par la transformation de l'apparence physique dans une logique d'uniformisation et de perte de repères. D'après nos entretiens, la coupe de cheveux est souvent redoutée. Certains appelés anticipent ce moment en allant chez le coiffeur avant même de se rendre à la caserne, mais l'institution les obligera de toute façon à se recouper les cheveux. Les barbes et les moustaches sont formellement proscrits dans l'institution. Les particularités et les signes distinctifs seront ainsi supprimés, comme les cheveux longs, les piercings, etc. Ensuite, les appelés seront soumis en petit groupe à un contrôle médical, qui suppose que l'appelé soit nu devant les autres. L'inoculation de deux ou trois vaccins, souvent effectuée en toute hâte d'après les témoignages, n'est pas expliquée aux appelés. Après cette étape, l'appelé devra 
Kaya, Sümbül (2009) 'La fabrique du 'soldat-citoyen' à travers la conscription en Turquie', European Journal of Turkish Studies, Thematic Issue $\mathrm{N}^{\circ} 8$, No. 8 | Surveiller, normaliser, réprimer, URL : http://www.ejts.org/document2922.html To quote a passage, use paragraph (§).

revêtir l'uniforme ainsi que les bottes qui sont distribuées sans tenir compte de la taille réelle des pieds des individus. Les entretiens effectués nous indiquent que les appelés ont le sentiment de ne plus se reconnaître eux-mêmes une fois que l'uniforme est vêtu et que les cheveux sont coupés. Certains précisent qu'ils avaient également du mal à distinguer leurs collègues des uns des autres car tous se ressemblaient. Ces pratiques sont révélatrices d'une atteinte à l'identité mais aussi d'un processus de dépersonnalisation qui atteint directement la représentation de soi et des autres des appelés. De plus, ces derniers ont un sentiment de désordre pendant que s'effectuent ces rites d'admission au sein de la caserne : le bruit, les cris, les odeurs de pied, les uniformes et bottes distribués à la hâte. Ils sont nombreux à avoir vu écrit ou entendu 'Bienvenue aux enfers' ou 'Bienvenue en enfer, vous n'êtes plus dans les bras de votre mère'. La pratique de la circoncision est proposée par l'institution. Elle est également une atteinte à l'intégrité physique de la personne, légitimée par un discours d'égal accès des pauvres à cette pratique. Soulignons à ce titre que dans les faits, la dimension religieuse permet paradoxalement de renforcer le processus de normalisation des corps au sein d'une institution militaire en principe garante de la laïcité.

[12] L'usage de la violence morale est également une pratique dont témoigne la majorité des entretiens. Les insultes et, plus généralement, le langage vulgaire utilisé par les supérieurs hiérarchiques participent de cette forme de dégradation de l'estime de soi. Les appelés intériorisent ainsi beaucoup plus rapidement l'idée de hiérarchie et leur position de dominés. D'ailleurs, les dominants n'hésitent pas à accentuer l'infantilisation des appelés par certaines postures physiques comme par exemple en ordonnant à l'appelé de ramper jusqu'à un arbre et de le saluer. Cette intériorisation est tellement forte que le garde-à-vous, ponctué par l'expression 'à vos ordres mon commandant' (Emret Komutanım), est effectué par le nouvel arrivant devant toute personne par peur de commettre une imprudence à l'égard de la hiérarchie.

[13] La violation du domaine de l'intime contribue également au processus d'intégration, comme dans toutes les institutions totales: comme l'exprime Goffman dans un autre contexte, 'les secteurs de la vie personnelle sont profanés' (Goffman 1968: 66). Pour l'auteur, il s'agit d'une contamination physique qui vise à mortifier l'individu, ce qui se traduit au sein de la caserne par une multitude de relations imposées. Effectivement, l'appelé, une fois intégré au sein de l'institution, ne se retrouvera plus jamais seul, sauf s'il est de garde. Pendant les classes, du dortoir aux entraînements, les appelés seront tout le temps intégrés dans des groupes. II est par ailleurs interdit de posséder des photos de famille, de tenir un journal intime dans certaines régions, et il est possible 
Kaya, Sümbül (2009) 'La fabrique du 'soldat-citoyen' à travers la conscription en Turquie', European Journal of Turkish Studies, Thematic Issue $\mathrm{N}^{\circ} 8$, No. 8 | Surveiller, normaliser, réprimer, URL : http://www.ejts.org/document2922.html To quote a passage, use paragraph (§).

d'être soumis à une enquête de moralité si l'institution souhaite confier à l'appelé des fonctions de renseignement ou de protection. L'institution militaire organise également un contrôle non hiérarchique par 'le système du buddy' 11 : le soldat a un binôme au sujet duquel il connaît tous les problèmes et préoccupations, renseignements sur sa famille... jusqu'au numéro de son arme. II doit également avertir le commandant si son binôme ne va pas bien et le buddy (badi) est tenu pour personnellement responsable de ce qu'il adviendra de l'autre, notamment en cas de suicide ou de désertion.

[14] L'institution de différentes pratiques comme l'isolement, l'uniformisation et la mortification semblent donc permettre la mise en condition de l'appelé afin de l'assujettir au moyen de dispositifs disciplinaires singuliers.

\section{Les dispositifs disciplinaires et la production d'un ethos militaire : des outils indispensables} à l'inculcation d'un imaginaire politique ?

[15] L'armée a pour objectif d'éduquer les appelés à la 'chose' militaire mais nous verrons plus bas qu'elle joue un rôle important au niveau de la socialisation politique des appelés et plus précisément dans la réactivation d'un certain l'habitus national. Les dispositifs disciplinaires visent-ils à faire intérioriser aux appelés un ethos militaire12 qui favorisera à son tour la réactivation d'un habitus national ? Nous retiendrons une définition de la discipline qui ne se réduit pas à l'aspect purement militaire et qui est celle définie par Michel Foucault comme 'des systèmes de surveillance continuelle et hiérarchisée' (Foucault 2001: 392) sur le corps qui permettrait l'imposition d'un rapport de 'docilité-utilité'. Les instruments de ce 'pouvoir disciplinaire' sont 'le regard hiérarchique, la sanction normalisatrice et leur combinaison dans une procédure qui lui est spécifique, l'examen' (Foucault 1975: 200). Nous allons donc décrire ces dispositifs militaires formels et informels en distinguant les différentes étapes du processus de normalisation disciplinaire13 qui est à l'œuvre au

${ }^{11}$ Buddy signifie dans le langage familier aux Etats-Unis copain ou pote. Le 'Buddy system' signifie 'par équipe de deux'.

${ }^{12}$ Nous entendons par ethos militaire un ensemble de dispositions militaires acquises pendant le service militaire. Bourdieu désigne par l'ethos 'un ensemble objectivement systématique de dispositions à dimension éthique, de principes pratiques'. Pour Bourdieu, 'la force de l'ethos, c'est que c'est une morale devenue hexis, geste, posture' (Bourdieu 1978 : 133-136).

${ }^{13}$ Michel Foucault définit la normalisation disciplinaire de la manière suivante : 'la normalisation disciplinaire consiste à poser d'abord un modèle, un modèle optimal qui est construit en fonction d'un 
Kaya, Sümbül (2009) 'La fabrique du 'soldat-citoyen' à travers la conscription en Turquie', European Journal of Turkish Studies, Thematic Issue $\mathrm{N}^{\circ} 8$, No. 8 | Surveiller, normaliser, réprimer, URL : http://www.ejts.org/document2922.html To quote a passage, use paragraph (§).

sein de l'institution militaire : la normalisation par l'examen, la normalisation par l'emploi du temps, la normalisation par la surveillance, la normalisation par le système gratification/coercition/violence.

[16] Dès la convocation de la personne, avant même son départ, à la division du service militaire (Askerlik Şubesi), l'examen de son profil (taille, corpulence, antécédents médicaux et psychologiques, formations et niveaux d'études...), conditionnera son affectation dans certaines brigades et régiments, mais définira aussi s'il est affecté à l'armée de terre, à la marine, ou à l'armée de l'air (nous y reviendrons). L'institution militaire effectue une classification/sélection au sein des différents groupes de conscrits dans chaque département.14 Les appelés officiers de réserve passent en plus un examen écrit qui déterminera la durée du service et le lieu d'affectation. Les simples soldats qui souhaitent devenir sergent par exemple passeront également un examen écrit à la fin de leur classe. Du début à la fin de son service militaire, l'appelé sera soumis à des examens multiples. Lors des classes, l'assimilation des techniques militaires sera également évaluée, voire notée, par exemple au niveau des tirs, mais aussi au niveau des entraînements et autres exercices physiques. A la fin des classes, l'appelé prêtera serment lors d'une cérémonie (yemin töreni). II s'entraînera tout au long de ses classes à défiler pour ce qui sera sa première exposition en public. Les parents ainsi que les proches sont conviés à cette cérémonie, car ce jour-là l'appelé devient un véritable soldat et portera une arme. C'est un moment important qui symbolise la fin des classes et les premières sorties de l'appelé en dehors de la caserne. Les appelés qui n'ont pas intégré leur formation de base (marches militaires, posture corporelle) ne défileront pas, ou seront présents mais cachés derrière les autres. Les appelés 'pourris' ou 'pedereme', qui ont des problèmes psychologiques, seront quand même maintenus au sein de la caserne mais sans responsabilités, ni accès aux armes. Sans être exclus de l'institution, ces derniers resteront en marge, en retrait, mais seront soumis comme les autres au processus de normalisation disciplinaire.

[17] L'emploi du temps permet par ailleurs de situer avec précision où se trouve chaque appelé, il s'agit d'un réglage très minutieux de l'espace-temps. La normalisation disciplinaire s'opère

certain résultat, et l'opération de la normalisation disciplinaire consiste à essayer de rendre les gens, les gestes, les actes conformes à ce modèle, le normal étant précisément ce qui est capable de se conformer à cette norme et l'anormal, ce qui n'en est pas capable. En d'autres termes, ce qui est fondamental et premier dans la normalisation disciplinaire, ce n'est pas le normal et l'anormal, c'est la norme'. (Foucault $2004: 59$ ).

${ }^{14}$ Pour Foucault, l'examen permet 'de qualifier, de classer et de punir' en combinant 'les techniques de la hiérarchie qui surveille et celle de la sanction qui normalise' (Foucault $1975: 217$ ). 
Kaya, Sümbül (2009) 'La fabrique du 'soldat-citoyen' à travers la conscription en Turquie', European Journal of Turkish Studies, Thematic Issue $\mathrm{N}^{\circ} 8$, No. 8 | Surveiller, normaliser, réprimer, URL : http://www.ejts.org/document2922.html To quote a passage, use paragraph (§).

également par la gestion de l'emploi du temps des appelés. Les jours se rythment de la même manière tout au long des classes. La journée débute à 5 heures du matin. Le gardien (nöbetçi) appelle le dortoir à se réveiller. Les appelés doivent faire leur lit au carré, leur toilette et se raser. A 6 heures, les soldats sont appelés à quitter le dortoir. Ils se regroupent dans la cour et doivent la nettoyer. A 6h30, les petits Mehmet - mehmetçik, nom générique et affectueux donné aux soldats attendent en rang dans la cour l'arrivée du commandant. Ils font une prière avant de manger : 'merci à notre Dieu que notre nation existe' (Tanrımıza hamdolsun milletimiz var olsun). Ensuite, ils se regroupent à nouveau dans la cour pour le contrôle vestimentaire et l'appel. A partir de 8 heures, les cours d'éducation militaire et les entraînements débutent, en quatre séquences de 45 minutes (avec 15 minutes de pause entre chaque cours). A midi, c'est à nouveau le rassemblement pour le repas. L'après-midi, les entraînements sont organisés en trois ou quatre séquences de 45 minutes. Certains cours comme les cours de formation à la citoyenneté ont lieu le soir, après le repas. Les soldats ne se coucheront qu'à 22 heures, le rythme est donc très intense pendant les classes.

[18] L'institution militaire est également dotée d'un système de surveillance formel et informel. L'attribution d'une place précise facilitera la surveillance des appelés. A l'arrivée de l'appelé au sein de la caserne, on lui attribue une fonction dans un peloton et une place dans une équipe15. On lui attribue également un numéro qui le positionne par rapport aux autres de son équipe et ceci déterminera la place qu'il occupera dans le dortoir tout au long de ses classes et pendant le reste du service. L'attribution d'une place précise facilite la surveillance en réseau. De plus, l'appelé doit se présenter matin et soir à des contrôles de présence. L'appelé se placera pendant le comptage dans un ordre déterminé par son numéro et l'équipe à laquelle il appartient.

[19] L'institution militaire s'est dotée d'un système de surveillance qui fonctionne de manière très similaire aux dispositifs décrits par Michel Foucault, c'est-à-dire en 'réseau de relations de haut en bas, mais aussi jusqu'à un certain point de bas en haut et latéralement; ce réseau fait ' tenir' l'ensemble, et le traverse intégralement d'effets de pouvoir qui prennent appui les uns sur les autres : surveillants perpétuellement surveillés' (Foucault 2001 : 208). Le pouvoir se diffuse à travers les corps, et les appelés - surveillés par leurs supérieurs hiérarchiques - se surveillent entre eux. Les appelés en Turquie sont soumis à toute une hiérarchie (d'officiers et de sous-officiers) qui est fixée par les grades, mais ils sont également et peut-être surtout soumis au 'système de période'. $\mathrm{Ce}$

${ }^{15}$ Chaque appelé est affecté à : un peloton (manga), une équipe (takım), un escadron ou compagnie (bölük), un bataillon (tabur), un régiment (alay), une brigade (tugay). 
Kaya, Sümbül (2009) 'La fabrique du 'soldat-citoyen' à travers la conscription en Turquie', European Journal of Turkish Studies, Thematic Issue $\mathrm{N}^{\circ} 8$, No. 8 | Surveiller, normaliser, réprimer, URL : http://www.ejts.org/document2922.html To quote a passage, use paragraph (§).

système, devrecilik sistemi, est une sous-hiérarchie organisée de manière informelle entre les appelés en fonction de leur ordre d'arrivée à la caserne : dede (grand-père), üst-devre (la période supérieure) ou kral devre (le période royale), torun (le petit-fils), pic torun (le petit-fils bâtard), torun (petit-fils) ou çömez (apprenti). Les autorités militaires nient formellement l'existence de cette hiérarchie informelle mais la tolèrent en réalité puisque ce système où les appelés s'autorégulent entre eux leur apporte également des bénéfices au niveau du maintien de la paix sociale. Cette hiérarchie officieuse connaît ses propres codes et règles. Les petits-fils, nom donné au primo arrivant à la caserne, sont soumis à un véritable 'bizutage' de la part des plus anciens. Ils devront effectuer toutes les tâches considérées comme dégradantes que les autres ne souhaitent pas faire - comme, par exemple le ménage - et généralement tout ce qui renvoie aux rôles féminins traditionnels. Cette 'féminisation' des primo arrivants au sein de l'institution est vécue comme dégradante par rapport à l'image dominante de la virilité chez de jeunes hommes, qui voient ainsi le renversement de leur position 'naturelle' au sein de la famille. 'Le petit-fils' accomplira toutes les tâches que les autres ne souhaitent pas faire, il ne participera pas aux prises de décisions même minime soit-elle comme, par exemple, le choix d'une chaîne de télévision. Lors d'un entretien, l'enquêté m'explique que, dans le système de période, "la période supérieure est la période royale, on est comme le commandant. [L'appelé de la période inférieure] fait ce qu'il dit, il est tapé, insulté. II doit faire ses gardes à sa place s'il le demande. Tu ne peux rien dire. Il est devenu le loup de là-bas, l'homme est là-bas depuis neuf mois et il connaît tout. Et toi, tu ne peux rien dire'16. Ce système de surveillance informel peut être considéré comme un dispositif très contraignant et dont le pouvoir normalisateur est assez conséquent sur les appelés. Les relations entre les gradés et les soldats de base sont en effet socialement très pauvres alors que la hiérarchie officieuse s'impose davantage au quotidien de l'appelé, qui interagit bien plus fréquemment avec les autres appelés de base appartenant à une période supérieure. En ce sens, cette hiérarchie officieuse ne remet pas en cause la hiérarchie officielle de l'armée mais la complète.

[20] L'appelé est ainsi soumis à une surveillance continue et nous observerons qu'il est également soumis à une 'pénalité perpétuelle' ou à une 'sanction normalisatrice' en cas de non respect de la discipline17. II sera sanctionné s'il est en retard, s'il n'exécute pas correctement les

${ }^{16}$ Entretien réalisé à Kayseri le 23 juillet 2007

17 Notons toutefois qu'en cas de violation du règlement intérieur existe un dispositif de sanctions juridiques précisé par l'article 2 du code pénal militaire, qui stipule également que 'le soldat doit 
Kaya, Sümbül (2009) 'La fabrique du 'soldat-citoyen' à travers la conscription en Turquie', European Journal of Turkish Studies, Thematic Issue $\mathrm{N}^{\circ} 8$, No. 8 | Surveiller, normaliser, réprimer, URL : http://www.ejts.org/document2922.html To quote a passage, use paragraph (§).

gestes, s'il échoue aux entraînements, s'il désobéit...Ces sanctions sont complétées par un système de gratification des appelés. Les prouesses sportives des soldats, leurs résultats au tir, leur capacité d'endurance pourront leur permettre d'obtenir une certaine reconnaissance des autres appelés mais aussi de leurs supérieurs hiérarchiques. Des certificats de bonne conduite et des documents d'appréciation sont également donnés aux appelés les plus méritants et surtout les plus respectueux de l'institution militaire. Cette valorisation des appelés correspondant au modèle du bon soldat constitue un instrument de normalisation par la sanction positive. La sanction devenue système ne vise pas simplement la répression, mais elle permet également de normaliser en différenciant, en hiérarchisant, en homogénéisant et en excluant, comme le souligne Foucault. L'institution militaire use donc de ces sanctions pour atteindre son objectif de 'normalisation disciplinaire'.

[21] Le recours à la violence physique et morale par les supérieurs hiérarchiques fait enfin partie intégrante de la sanction normalisatrice au sein de l'institution militaire turque. Dès l'arrivée de l'appelé, la violence physique et symbolique est omniprésente au sein de la caserne, de sorte que l'appelé peut sentir son intégrité physique menacée. De nombreux enquêtés confirment cette violence : 'celui qui dit n'avoir pas été battu pendant son service est un menteur'18. La majorité des enquêtés, qui étaient de simples soldats, expliquent avoir été victimes de violences dont l'intensité varie selon les situations. Les sanctions corporelles sont en général des claques et des coups de pied, parfois de véritables passages à tabac. Ce sont souvent des sanctions exemplaires qui visent à dissuader les autres de commettre la même erreur. Les causes sont diverses : manquement à un ordre, cheveux trop longs, mauvaise gestuelle ou position militaire inadéquate. Les sanctions sont également humiliantes ou infantilisantes comme par exemple lorsqu'un appelé est obligé de saluer un arbre un certain nombre de fois. Les sanctions collectives sont également très fréquentes. Du fait de l'erreur de l'un, les autres peuvent être soumis à des exercices collectifs ou être interdits de sortie en fin de semaine. II en résulte des comportements violents entre appelés eux-mêmes, notamment lorsque le système des périodes n'est pas respecté. Ahmet est professeur de mathématique, il a été officier de réserve et m'a soigneusement décrit comment il avait géré un problème avec un appelé. :

contribuer, apprendre et protéger la nation turque, l'indépendance et la république... et pendant son service il doit porter l'uniforme. Les appelés qui commettent des infractions au règlement intérieur seront jugés selon le code pénal militaire'. Askeri Ceza Kanunu, n¹632, adopté le 22/05/1930.

${ }^{18}$ Entretien avec Volkan, le 12 avril 2006 à Kayseri, mais cette phrase revient dans plusieurs entretiens. 
Kaya, Sümbül (2009) 'La fabrique du 'soldat-citoyen' à travers la conscription en Turquie', European Journal of Turkish Studies, Thematic Issue $\mathrm{N}^{\circ} 8$, No. 8 | Surveiller, normaliser, réprimer, URL : http://www.ejts.org/document2922.html To quote a passage, use paragraph (§).

J'étais avec un sous-officier et moi j'étais officier. J'ai fait appeler ce jeune. II s'est assis et je lui ai demandé lequel d'entre nous était le plus gradé. II m'a répondu que le plus gradé était le sous-lieutenant car il l'avait frappé beaucoup plus que moi. Ce jeune appelé avait des poux sous le bras. J'ai regardé et il avait des poux. J'ai fait ramener son lit et ses affaires personnelles à l'extérieur. On lui a demandé de se mettre tout nu. Un natif de Tunceli m'a dit qu'il ne se lavait pas. J'ai constaté que ses deux sous-vêtements étaient tout noirs. II était de Sinop. J'ai brûlé toutes ses affaires. Les soldats étaient autour de lui. J'ai ordonné qu'on le lave et qu'on le rase et je lui ai donné des vêtements propres qui m'appartenaient, en enlevant les galons. En fait, ce jeune était retardé, un peu débile. À la fin je lui ai dit : si j'entends un truc sur toi je te jetterai dans la rivière et tes parents ne te retrouveront plus jamais'19.

[22] Ce discours illustre bien le degré d'intensité que peut prendre la violence morale. II s'agit également d'une sanction exemplaire qui vise à dissuader les autres appelés en diffusant la crainte d'être confronté à une même situation. L'officier de réserve réaffirme sa position en diffusant une image paternaliste de lui-même : en lui donnant son uniforme, en le mettant en garde avant de recourir potentiellement à la violence physique.

[23] La répétition des mêmes entraînements militaires et l'apprentissage des positions de bases encadrées par des dispositifs disciplinaires visent donc l'incorporation par les appelés d'un ethos militaire systématique. En effet, certaines transformations peuvent être constatées chez les personnes ayant effectué leur service militaire : le rythme et l'intensité des entraînements modifient les corps des appelés, sauf s'ils ont été dispensés d'entrainements et de sport, et les postures militaires impliquent une certaine droiture et rigidité des mouvements, visible chez les jeunes sortis récemment de l'institution. De même, nos entretiens effectués avec un appelé avant et juste après son service militaire révèlent des manières de s'exprimer très marquées par l'institution (caractère plus audible des propos, etc.). Ces modifications corporelles peuvent bien sûr s'estomper lors du retour à la vie civile et ne sont pas forcément durables puisque l'arrêt des entraînements permet de retrouver son 'hexis corporelle' d'origine, jusqu'à ce qu'une nouvelle situation puisse éventuellement les activer à nouveau. En revanche, certaines dispositions au niveau cognitif transforment beaucoup plus durablement, nous semble-t-il, les dispositions des appelés : il s'agit du respect de la hiérarchie et de la soumission à l'autorité. Tout se passe en fait comme si l'intégration de l'appelé au sein des

\footnotetext{
${ }^{19}$ Entretien avec Ahmet, le 25 mars 2006 à Kayseri.
} 
Kaya, Sümbül (2009) 'La fabrique du 'soldat-citoyen' à travers la conscription en Turquie', European Journal of Turkish Studies, Thematic Issue $\mathrm{N}^{\circ} 8$, No. 8 | Surveiller, normaliser, réprimer, URL : http://www.ejts.org/document2922.html To quote a passage, use paragraph (§).

forces armées et sa soumission aux dispositifs disciplinaires visaient in fine la formation d'un citoyen turc conforme au projet politique de l'Etat kémaliste.

\section{La réactivation d'un habitus national conforme à l'idéologie kémaliste de l'Etat Turc ?}

[24] Les dispositifs disciplinaires ont donc préparé les corps des appelés qui deviennent 'utiles et dociles', ce qui permettra à l'institution de se consacrer à la formation de leur esprit. En Turquie, l'armée revendique le fait d'être une école pour la nation (Ünsaldı 2005). Un entretien avec un commandant de gendarmerie est éclairant sur ce point : 'En dehors de la défense de la patrie, l'importance du service est de cultiver, éduquer le peuple, la société. L'une des missions de l'armée est de discipliner la société ... II y a des appelés qui ne savent pas lire, même pas lire l'heure, ils ne savent même pas utiliser les toilettes'20. Ce discours reprend le mode central de légitimation de la bourgeoisie turque depuis le 19ième siècle, à savoir l'opposition entre élites connaissantes et peuple ignorant. Par ailleurs, cette 'école de la nation' peut aussi faire intérioriser aux appelés une certaine conception de l'identité nationale. Nobert Elias souligne l'importance du rôle des institutions et de l'éducation dans 'l'approfondissement et le renforcement d'un sentiment du 'nous' exclusivement axé sur la tradition nationale' (Elias 1991 : 273 \& Elias 1989). Selon l'auteur, les institutions garantissent que différentes personnes au sein d'une même société acquièrent certaines caractéristiques communes, c'est-à-dire des éléments d'un habitus national21. Nous allons donc analyser à travers la formation des appelés comment peuvent se renforcer et se réactiver les dispositions relatives à 'l'identité collective nationale'. Nous allons décrire la 'formation à la citoyenneté' (Vatanda lık E itimi) qui est dispensée par l'institution militaire aux appelés pendant leur service et monter qu'elle est cohérente à la conception de l'historiographie nationale diffusée à l'école. Ce qui nous conduira à soutenir l'idée que cette formation réactive l'habitus national, sans doute de façon relativement durable.

[25] La 'formation à l'amour de la patrie' (Yurt Sevgisi E itimi), renommée en 2000 'formation à la citoyenneté' (Vatanda lık E itimi), est dispensée à tous les appelés. Son existence montre que l'institution militaire joue un rôle important dans l'entretien de l'idéologie officielle kémaliste. Pour Etienne Copeaux, le 'consensus obligatoire' est l'ensemble de jugements, de

20 Entretien réalisé le 12 juin 2007 à Kayseri.

${ }^{21}$ Voir la note 3. 
Kaya, Sümbül (2009) 'La fabrique du 'soldat-citoyen' à travers la conscription en Turquie', European Journal of Turkish Studies, Thematic Issue $\mathrm{N}^{\circ} 8$, No. 8 | Surveiller, normaliser, réprimer, URL : http://www.ejts.org/document2922.html To quote a passage, use paragraph (§).

comportements publics exigés du citoyen' en Turquie (Copeaux 2000: 89). L'institution militaire semble donc être un instrument important de réaffirmation de ces normes et de vérification de la bonne intériorisation de l'idéologie kémaliste.

[26] Les documents internes aux forces armées turques22 soulignent que le but de ces formations à la citoyenneté est de montrer 'le vrai visage' de l'ennemi et de mettre en garde les jeunes Turcs contre les trois menaces que constituent la réaction politique (irtica), le séparatisme (bölücülük), et l'immoralité (ahlaksızlık). C'est pourquoi, il est nécessaire 'de créer un environnement propice au développement d'une relation de confiance et d'un lien respectueux envers les forces armées turques et envers le personnel militaire'. II ressort également de ce projet que, pour protéger l'avenir de la Turquie, il est indispensable de mettre en œuvre les formations nécessaires en accord avec la pensée d'Atatürk : 'la caserne n'est pas seulement le lieu d'apprentissage de la guerre, elle est en même temps le foyer d'une culture, elle est l'école de l'art et en agissant ainsi le service rendu à la nation est incommensurable'23. Le service militaire est également considéré comme 'une valeur sacrée pour les Turcs': 'chaque Turc quand le moment arrive et avec la fierté que donne le fait d'être soldat accomplira sa fonction avec amour. A la caserne à côté de l'apprentissage de l'art militaire, il sera donné des cours relatifs à la vie afin de préparer les jeunes à la vie. C'est pourquoi lorsque l'appelé se rend à son service, il s'y rend avec amour (...) C'est quelque chose qu'on n'a jamais vu chez les autres nations. Les jeunes Turcs partent faire leur service et la guerre comme s'ils allaient à leur cérémonie de mariage, et cette totalité armée-nation est le plus bel exemple de cet amour'24. Quand l'appelé prête serment lors de la cérémonie, il doit répéter le discours suivant : 'En période de guerre ou de paix, en mer et dans les airs, à tout moment et partout, avec amour et droiture, je rendrai service à ma Patrie et à ma République, j'obéirai aux lois et aux ordres et aux commandants et je saurai que le drapeau Turc et l'honneur du service sont plus sacrés que ma vie et je sacrifierai ma vie avec amour dans le but de ma fonction et pour ma Patrie et ma République...'25.

[27] Les documents internes destinés au personnel enseignant chargé de la formation à la citoyenneté, mettent également en évidence les normes conformes à l'idéologie officielle de l'Etat

22 Yurt Sevgisi Bilinci Kazandırma Eğitimi, Projet de développement des connaissances d'amour de la patrie.

${ }^{23}$ Ibid.

${ }^{24}$ Notes de cours pour le personnel enseignant de la caserne.

${ }^{25}$ Eğitim Broşürü, le petit livre du soldat. 
Kaya, Sümbül (2009) 'La fabrique du 'soldat-citoyen' à travers la conscription en Turquie', European Journal of Turkish Studies, Thematic Issue $\mathrm{N}^{\circ} 8$, No. 8 | Surveiller, normaliser, réprimer, URL : http://www.ejts.org/document2922.html To quote a passage, use paragraph (§).

turc. Les citations d'Atatürk sont d'ailleurs très nombreuses tout au long des cours. Ces derniers sont divisés en deux parties : la première s'intitule 'Acquisition et développement de la citoyenneté' et la deuxième 'renforcer le sentiment de citoyenneté'. La première partie explique la formation de l'Etat turc à partir des principes de base de la république et du système de pensée d'Atatürk26. Dans les documents power point qui accompagnent ces textes 27 , il est précisément indiqué que chaque jeune Turc sera instruit au sein des institutions éducatives turques à la lumière de la pensée d'Atatürk. Le nationalisme d'Atatürk est également expliqué par opposition à ce qu'il n'est pas, c'est-à-dire aux idéologies de race, de classe, de communauté, de région, de division. Le nationalisme d'Atatürk est défini comme 'une conscience culturelle et la chaleur d'un sentiment'. La vie d'Atatürk est longuement mise en avant. La finalité de l'institution est de faire intérioriser, notamment en apprenant par cœur, le système de pensée d'Atatürk. Aussi, dans la première partie du cours intitulé 'Acquisition et développement de la citoyenneté', des thèmes très variés sont abordés, des droits et devoirs de l'Etat et du citoyen jusqu'aux règles de conduite automobile28. Dans la seconde partie intitulée 'renforcer le sentiment de citoyenneté', l'appelé est censé développer ses connaissances sur les problèmes et les menaces qui pèsent sur la Turquie. II aborde ainsi l'histoire de la Turquie, sa situation géopolitique, la sécurité nationale et le pouvoir national (la force militaire, l'objectif national, l'intérêt national)29. L'institution propose aux appelés un modèle d'humanisme, d'où l'insistance sur

${ }^{26}$ Atatürk et l'unité nationale, Atatürk et la civilisation, la compréhension de la démocratie par Atatürk, Atatürk et la justice, la conception de la nation par Atatürk, la conception de la culture par Atatürk, la conception de la nationalité par Atatürk, la suppression du sultanat et sa conception du peuple, l'analyse de l'Etat par Atatürk, les analyses contemporaines d'Atatürk, les guerres auxquelles a participé Atatürk, les conseils et recommandations d'Atatürk dont un passage sur le service militaire et l'armée, la compréhension de la République par Atatürk, que se serait-il passé si Atatürk n'avait pas été là.

${ }^{27}$ YSE Ĕgitici Ĕgitimi, Formation à l'amour de la patrie et formation aux formateurs.

${ }^{28}$ La deuxième sous-partie est consacrée à l'apprentissage et au développement des données sur la citoyenneté : les devoirs de l'Etat envers le citoyen, les devoirs économiques et sociaux, les devoirs du citoyen envers l'Etat (payer des impôts, faire son service militaire, respecter les lois et les règlements, être dans une attitude et pensée solidaire, attachement au peuple, attachement à l'Etat, attachement au citoyen, attachement aux lois, s'approprier et défendre la république), les règles de conduite automobile, l'économie-épargne, les problèmes environnementaux (protection de l'environnement et catastrophe naturelle). La troisième sous-partie est consacrée au développement et renforcement des valeurs de base et se divise ainsi : les valeurs universelles, le respect de la vérité, la plénitude individuelle, l'équité, la dignité de l'individu, rendre service et l'entraide.

${ }^{29}$ Dans les cours d'histoire, l'origine du peuple turc est longuement expliquée puis sont évoqués plus loin les rapports de la Turquie avec la Grèce, le question des minorités, les activités destructrices et 
Kaya, Sümbül (2009) 'La fabrique du 'soldat-citoyen' à travers la conscription en Turquie', European Journal of Turkish Studies, Thematic Issue $\mathrm{N}^{\circ} 8$, No. 8 | Surveiller, normaliser, réprimer, URL : http://www.ejts.org/document2922.html To quote a passage, use paragraph (§).

les valeurs morales et les comportements en dehors même de l'institution. Les valeurs éthiques et morales du citoyen caractérisent l'homme turc idéal : l'honnêteté, la détermination et la résistance, la patience et le courage, le culte du secret, les ambitions et les idées communes, l'entraide et l'apparence soignée. Une partie est également consacrée à la conception de la vie de la famille et de la société30. Puis, les questions concernant la culture nationale31 et les valeurs influençant la société turque sont abordées32, renforçant ainsi le sentiment d'unité nationale. L'ambition homogénéisatrice de l'institution militaire est clairement mise en évidence dans ces documents internes. Le « bon citoyen » est également décrit comme fidèle, respectueux, honnête et courageux, possédant le sens du devoir et celui de l'honneur, sachant rendre service avec abnégation. Le renforcement du « nous », de l'identité collective nationale, prend donc appui sur ces caractéristiques de l'identité individuelle que le soldat-citoyen doit intégrer.

[28] Or, l'analyse des normes véhiculées par l'école à travers notamment les manuels d'histoire (Copeaux 1997) montre une congruence avec celles développée par l'institution militaire: les secondes visent donc très largement à renforcer les premières. Si ce phénomène n'est pas une spécificité turque, certains facteurs le rendent peut-être ici plus probant que dans d'autres pays. En opérant une continuité stricte entre socialisation primaire et secondaire, ces deux institutions véhiculent en effet les mêmes normes conformes à l'idéologie kémaliste : cette 'éducation totale' à travers l'école et la caserne (Faguer 1991: 25-43) tend à assurer l'ancrage et réactivation d'un l'habitus national (conception de l'Etat-nation en Turquie, valeurs morales et éthiques qualifiées de nationales, etc.) chez les appelés. Ces dispositions ont été comme le suggère Bernard Lahire 'mises

séparatistes, le terrorisme, les missionnaires en Turquie, les stratégies et buts de l'islam politique, les ONG, les irrégularités et détériorations culturelles, ce que ne fait pas un bon citoyen, les menaces extérieures, les conflits asymétriques, le lien social et la cohésion nationale. Une autre partie est consacrée au principe de sécurité nationale et au pouvoir national avec une explication de la notion de sécurité nationale, aux menaces et aux nécessités de sécurité nationale, au système de sécurité nationale, à la force militaire...

${ }^{30}$ Renforcement et développement des informations sur le rôle de la morale et de l'éthique sur la vie de la famille et de la société : la famille et la compréhension de la famille turque (l'éducation de la famille, le planning familial, la contraception, le mariage consanguin, les maladies sexuellement transmissibles, la communication au sein de la famille, l'usage de la force et des sanctions).

${ }^{31}$ Les peuples turcs et l'attachement à la culture turque, les traditions turques et leur importance, les opinions importantes du peuple turc comme l'amour de la patrie, l'amour du pays, l'amour du drapeau, l'amour du service militaire.

32 Les règles juridiques, les coutumes et traditions, les règles de savoir vivre, les règles morales, les facteurs religieux, la psychologie. 
Kaya, Sümbül (2009) 'La fabrique du 'soldat-citoyen' à travers la conscription en Turquie', European Journal of Turkish Studies, Thematic Issue $\mathrm{N}^{\circ} 8$, No. 8 | Surveiller, normaliser, réprimer, URL : http://www.ejts.org/document2922.html To quote a passage, use paragraph (§).

en attente... ou en suspens temporairement ou plus durablement' et 'leur remise en activité peut dépendre de la micro-situation sociale' (Lahire 2001 : 102). Les appelés soumis aux rites d'admission que nous avons décrits, coupés de leur environnement habituel, puis assujettis à des dispositifs disciplinaires sont désorientés. La fixation ou l'activation des structures de l'habitus national par l'institution peut prendre appui sur ce sentiment de perte de repères. De plus, la conjonction d'un conflit armé effectif entre l'armée turque et les guérillas du PKK où combattent déjà de nombreux appelés et du sentiment d'insécurité dû à la crainte d'une atteinte à l'identité nationale renforce l'ancrage individualisé de l'habitus national. Le fait d'impliquer directement ou indirectement les conscrits dans le conflit pendant leur expérience du service militaire renforce donc la fixation de l'habitus national. Gérard Noiriel soutient l'idée que le sentiment national peut être mobilisé par les individus dans certaines circonstances: 'Le fait que cette dimension identitaire soit en partie intériorisée sous forme d'habitus a pour conséquence qu'elle peut être facilement réactivée par ceux qui se présentent comme les porte parole de l'identité nationale (...) Mais les citoyens découvrent qu'il possède (ou qu'ils devraient posséder) une 'identité nationale' lorsque leurs intérêts sont 'menacés'...'(Noiriel 2001 : 138-139). II est également nécessaire de souligner que cette inculcation idéologique n'est pas seulement l'apanage de l'institution militaire et de ses professionnels, mais aussi des appelés qui peuvent veiller au bon respect de ces normes entre eux ce qui renvoie à la surveillance en réseau que nous avons déjà évoqué plus haut.

[29] Cependant, les observations et les entretiens avec les appelés nous montrent qu'ils ne correspondent pas tous au modèle du bon 'soldat-citoyen'. Effectivement, les enquêtés rencontrés nous ont fait part d'expériences très différentes qui pourraient sans doute être liées à la diversité de leurs expériences sociales antérieures et à la possession de capitaux sociaux, économiques et culturels singuliers: tout porte donc à croire que les effets et les usages de l'institution diffèrent fortement d'un individu à l'autre.

\section{Une homogénéisation très partielle : inégalités devant l'affectation et diversités des prises de rôle}

[30] Cette partie tente d'évaluer si les dispositifs disciplinaires et la formation à la citoyenneté produit des effets concrets à la hauteur de l'espérance de l'institution militaire. Certains appelés correspondent parfaitement au modèle du bon 'citoyen-soldat' et nous pouvons en déduire 
Kaya, Sümbül (2009) 'La fabrique du 'soldat-citoyen' à travers la conscription en Turquie', European Journal of Turkish Studies, Thematic Issue $\mathrm{N}^{\circ} 8$, No. 8 | Surveiller, normaliser, réprimer, URL : http://www.ejts.org/document2922.html To quote a passage, use paragraph (§).

que les objectifs de l'institution semblent souvent être atteints. Une majorité de nos enquêtés s'opposent ainsi à la surpression du service militaire et à la professionnalisation de l'armée turque. Ils considèrent le service militaire comme un devoir patriotique qui est nécessaire pour protéger la nation Turque. Pour Kadir qui est nationaliste : 'le service militaire est une dette. S'il y a une guerre, j'irai en courant. Si nous laissons cette mission aux professionnels l'armée s'affaiblira. De plus, l'armée turque ne peut payer tout le monde. Je ne vois pas la Turquie sans notre armée actuelle'33. Par ailleurs, certaines orientations politiques des appelés se rapprochent de la conception de l'identité nationale dont l'institution militaire fait la propagande. Effectivement Les militants ou sympathisants du Parti Républicain du Peuple (CHP) ou du Parti du mouvement national (MHP) possèdent une socialisation politique qui est déjà conforme à celle de l'institution. Dans ce cas, le passage par l'institution renforce les convictions politiques de ces appelés qui se conformeront aux normes de l'institution, presque indépendamment de l'action des dispositifs disciplinaires et de la formation militaire, mais aussi de leurs capitaux initiaux. II nous semble cependant que l'institution ne peut pas produire un ethos militaire uniformément docile à l'incorporation d'un habitus national homogène. La fonction qu'occupe chaque appelé peut d'abord varier en fonction des capitaux économiques, sociaux et culturels et donc produire des expériences différenciées, démontrant que les appelés sont soumis aux dispositifs disciplinaires et à la formation à la citoyenneté de manière très inégale. Indépendamment de ces fonctions attribuées par l'institution, d'autres facteurs comme l'âge d'incorporation et le niveau d'étude, les orientations politique et idéologique, les expériences et trajectoires sociales affectent ensuite la prise de rôle par les appelés au sein de l'institution. En effet, les appelés ont recours à des pratiques déviantes, d'accommodement, de distanciation, de résistance qui montrent que la tenue des rôles reste très hétérogène. Parmi ces pratiques, nous analyserons plus précisément celles qui visent à s'auto-disqualifier, à rompre l'isolement, à se libérer l'esprit, à éviter la confrontation avec les supérieurs hiérarchiques, à violer le monopole du commerce, et à se réfugier dans les croyances contraires à celle de l'institution.

[31] La diversité des expériences du service militaires s'explique d'abord par le fait que les fonctions attribuées par l'institution aux appelés varient selon les capitaux qu'ils possèdent. L'origine sociale conditionne en effet les lieux d'affectation, mais aussi les fonctions. Ainsi, plus l'appelé possède de capitaux économiques, sociaux et culturels, et moins il sera soumis à une surveillance

${ }^{33}$ Entretien réalisé le 2 mars 2006 à Kayseri. 
Kaya, Sümbül (2009) 'La fabrique du 'soldat-citoyen' à travers la conscription en Turquie', European Journal of Turkish Studies, Thematic Issue $\mathrm{N}^{\circ} 8$, No. 8 | Surveiller, normaliser, réprimer, URL : http://www.ejts.org/document2922.html To quote a passage, use paragraph (§).

continue, à des contraintes lourdes, à des violences extrêmes, alors même que l'institution prône l'égalité. Les hauts gradés n'oseront pas employer la violence par crainte d'un dépôt de plainte. De plus, certains militaires professionnels profiteront des avantages économiques, sociaux et culturels que peuvent leur procurer ces appelés, comme par exemple donner des cours de soutien scolaire aux enfants du commandant. Un de nos enquêtés était craint par son commandant car il était proche d'un haut gradé ce qui lui permettait d'avoir certaines libertés, comme sortir à son aise de la gendarmerie. La proximité sociale entre les appelés qui appartiennent à des classes sociales moyennes et supérieures et les militaires professionnels favorise également des rapports fondés beaucoup plus sur des liens affectifs que sur la coercition et l'autorité. En revanche, les dispositifs disciplinaires s'appliquent davantage aux classes populaires et aux classes moyennes inférieures. En effet, les commandos, qui sont souvent envoyés dans l'Est de la Turquie, sont recrutés dans ces catégories sociales. Souvent, il s'agit d'enfants de paysans qui connaissent des conditions de vie déjà difficiles dans la vie civile. Ces catégories sont en général plus surveillées et les appelés possédants davantage de capitaux économiques, sociaux et culturels sont chargés de les former. D'ailleurs, ces soldats du rang sont les cibles privilégiées des cours de développement de la citoyenneté. Deniz, professeur d'anglais à l'université, avait pour fonction pendant son service d'accueillir les délégations militaires étrangères. La description de son service montre qu'il ne vit pas reclus au sein de l'institution et qu'il multiplie les échanges dans le cadre de sa fonction avec des personnes extérieures : 'Comme je vis en civil, j’ai un appartement avec un ami à Istanbul. Je me promène souvent et je vais sur les îles à Istanbul. Je vais au théâtre, à l'opéra. Je n'ai aucune interdiction. Ces interdictions existent pour les soldats de base. Je n'ai pas le droit de sortir en dehors d'Istanbul'34. Les inégalités économiques et sociales inhérentes à la vie en dehors de la caserne sont largement reproduites à l'intérieur de la caserne. Deniz n'est donc pas soumis aux dispositifs disciplinaires habituels et à la formation à la citoyenneté.

[32] Le service n'est pas le même à l'Est (dans les zones de guérilla) ou à l'Ouest. De nombreux appelés ainsi que leurs proches appréhendent l'affectation dans l'Est car ils vont devoir combattre et dans tous les cas les conditions du service seront plus difficiles qu'à l'Ouest : difficulté à avoir une hygiène quotidienne, à manger, à dormir, à supporter les entraînements en montagne. D'ailleurs, les anciens combattants ne souhaitent plus parler de leur service. Ils souhaitent même,

${ }^{34}$ Entretien réalisé le 27 mars 2006 à Kayseri. 
Kaya, Sümbül (2009) 'La fabrique du 'soldat-citoyen' à travers la conscription en Turquie', European Journal of Turkish Studies, Thematic Issue $\mathrm{N}^{\circ} 8$, No. 8 | Surveiller, normaliser, réprimer, URL : http://www.ejts.org/document2922.html To quote a passage, use paragraph $(\S)$.

disent-ils, l'oublier35. Nous pouvons distinguer les jeunes qui ne veulent plus jamais parler de cette expérience (refoulement du traumatisme et silence) de ceux qui partageront pendant de longues heures avec d'autres leurs histoires du service tout en déformant ou exagérant certaines réalités pour amuser les autres ... Ziya a essayé de s'informer avant son départ dans le sud-est du pays, et il explique que 'certains me racontaient leur expérience dans le sud-est mais ce n'était pas vraiment la réalité. Si on se mettait à vraiment raconter ce qu'il se passe là-bas, plus personne ne voudrait partir faire son service'36. L'expérience de Halil qui est issu d'un milieu populaire nous montre que son service militaire a été réalisé dans des conditions difficiles, voire traumatisantes :

Le service est passé de 18 mois à 15 mois. Au lieu de le diminuer, on devrait le supprimer. Envoyer des appelés en opérations... Ça ne sert à rien. Un appelé ne pense qu'à rentrer et il ne pense qu'à la fin du service. Tu ne peux pas te sauver tu dois accomplir ta tâche, ta fonction. Mais, pendant les classes oui tu peux essayer de te sauver de tes fonctions de ne pas les accomplir. Dans l'Est, nous n'avions aucune activité de loisirs. On sortait avec les habits militaires. On n'avait pas d'activités de loisir. Je bougeais souvent, car j'étais chauffeur. Je suis parti 4 ou 5 fois en opération. Et je suis celui qui est le moins parti. Pour les autres, leurs mères pleuraient. Nous étions dans les montagnes. Nous n'avions pas de repas chaud ni de douche régulière. (...) J'ai essayé d'attraper un terroriste et de le tuer. Mais je n'ai pas réussi. Je voulais faire cela pour venger mon ami. C'est très dur, je voulais que mon service se termine vite. Je suis parti j'ai vite mis mes vêtements civils. Ils nous ont ramené à anllurfa et je me suis sauvé car j'ai préféré continuer mon chemin sans les autres militaires....37.

[33] II nous semble que les dispositifs disciplinaires ainsi que la formation à la citoyenneté s'imposent davantage sur les soldats de rang dans les régiments à l'Est. Ce contexte peut favoriser la réactivation de l'habitus national de façon plus homogène. L'identité nationale se renforce en distinguant les ennemis de la nation.

[34] Par ailleurs, d'autres facteurs peuvent influencer les appelés dans la tenue de leur rôle. II peut s'agir de l'âge d'incorporation, de l'origine sociale, culturelle et linguistique, de l'origine rurale ou urbaine, des orientations politiques et idéologiques, des expériences et les trajectoires sociales.

${ }^{35}$ Entretien avec un ancien combattant réalisé le 3 juillet 2008 à Kayseri.

${ }^{36}$ Entretien réalisé le 24 juillet 2007 à Kayseri.

37 Entretien réalisé le 15 mars 2006 à Kayseri. Afin de les protéger des attentats potentiels, l'institution militaire prévoit des bus pour aider les appelés à rentrer à leur domicile à la fin de leur service. 
Kaya, Sümbül (2009) 'La fabrique du 'soldat-citoyen' à travers la conscription en Turquie', European Journal of Turkish Studies, Thematic Issue $\mathrm{N}^{\circ} 8$, No. 8 | Surveiller, normaliser, réprimer, URL : http://www.ejts.org/document2922.html To quote a passage, use paragraph (§).

Nous n'analyserons ici à titre d'exemple que la question de l'âge, qui est intimement liée à celles des ressources. L'âge d'incorporation est en effet un facteur pouvant influencer la tenue du rôle. Plus les appelés sont âgés et plus leur identité personnelle est structurée et construite. Les jeunes hommes mettent en place des stratégies pour repousser la date de départ en s'inscrivant à des cours dans des instituts privés. Encore faut-t-il avoir les moyens économiques pour cela. Pour ceux qui ne feront pas d'études supérieures, il sera plus difficile de décaler le départ et donc ils accompliront ce devoir à l'âge de 19 ou 20 ans. En revanche, pour les personnes qui ont un niveau d'étude supérieur, il est davantage envisageable de retarder la date de départ. Ainsi, ils possèdent une expérience sociale qui est beaucoup plus longue que ceux qui ne possèdent pas de niveau d'études supérieures et ils ont donc accumulés davantage de ressources pour se protéger. En revanche, les jeunes qui ont terminé le lycée et n'ont pas fait d'études supérieures partent à 19 ans sous le drapeau. Leur identité est en train de se construire et on peut supposer que les composantes de l'habitus national structurent plus facilement cette identité. L'âge d'incorporation a assurément des conséquences sur le rapport à l'institution, sur le rapport à la discipline et à la hiérarchie... qui montre encore une fois que l'armée ne peut pas produire un ethos militaire uniforme et un habitus national homogène.

[35] Chaque appelé a différentes manières de tenir son rôle qui découle des différents facteurs que nous avons énoncé plus haut. Nos observations effectuées dans une gendarmerie nous ont permis de constater que les règles et normes ne sont pas toujours respectées. Les appelés n'étaient par exemple pas vêtus de la tenue vestimentaire réglementaire, ils fumaient alors que cette pratique est formellement interdite dans les chambrées... Erving Goffman suggère que ces 'adaptations secondaires' émaneraient de la tentative de l'individu de se distancier de l'institution et par conséquent qu'elles semblent être essentielles à la préservation du moi38. II découle de ces adaptations secondaires des pratiques destinées à se distancier du rôle que leur assigne l'institution et à échapper ainsi au regard hiérarchique, à la surveillance, à la discipline et à la propagande du kémalisme. La contestation et l'opposition à l'institution peuvent prendre des formes subtiles, 'ne laissant plus de prise à l'institution' (Bessin 2002 : 9). La préservation d'une partie de son intimité, de son moi intime face à l'emprise de l'institution, le refus du rôle assigné par l'institution et la prise de

38 Les 'adaptations secondaires' sont pour Goffman 'toute disposition habituelle permettant à l'individu d'utiliser des moyens défendus, ou de parvenir à des fins illicites (ou les deux à la fois) et de tourner ainsi les prétentions de l'organisation relatives à ce qu'il devrait faire ou recevoir, et partant à ce qu'il devrait être. Les adaptations secondaires représentent pour l'individu le moyen de s'écarter du rôle du personnage que l'institution lui assigne tout naturellement' (Goffman 1968 : 372). 
Kaya, Sümbül (2009) 'La fabrique du 'soldat-citoyen' à travers la conscription en Turquie', European Journal of Turkish Studies, Thematic Issue $\mathrm{N}^{\circ} 8$, No. 8 | Surveiller, normaliser, réprimer, URL : http://www.ejts.org/document2922.html To quote a passage, use paragraph (§).

distance se manifestent ainsi par le silence, l'apathie, l'indifférence, l'absentéisme, l'inactivité, ou les planques. Mais elle peut également être moins passive.

[36] Certains appelés sont stigmatisés par les autres et par l'institution comme 'fous' du fait qu'ils ont recours à des pratiques extrêmes comme le suicide, l'automutilation, la désertion, le nonrespect des règles et lois militaires. Les sanctions disciplinaires, voire le passage devant les tribunaux militaires, peuvent amener des peines d'emprisonnement ou même une prolongation du service militaire. L'institution militaire ne leur donne pas d'arme et les maintient au sein de la caserne, où ils n'endosseront pas de responsabilité. On peut également analyser les pratiques de ces appelés comme auto-disqualifiantes. L'institution qui en a besoin les présentera comme des contre-modèles. On peut retrouver dans cette catégorie des individus qui ont été soumis à d'autres socialisations politiques, culturelles et linguistiques. On peut retrouver dans cette catégorie à la fois des kurdes, des punks, des anarchistes ou des personnes ayant des troubles psychologiques...

[37] Certaines pratiques des appelés visent également à rompre l'isolement et la séparation avec le monde civil que l'institution leur impose, par exemple, par la détention d'un téléphone portable. Les appelés gardent ainsi le lien avec leurs proches et préservent une vie intime qui échappe à la logique de l'institution. L'invention d'une maladie ou d'un problème psychologique permet également à l'appelé d'éviter les entraînements ou d'obtenir une permission de sortie ce qui correspond aussi à une prise de distance avec son rôle de soldat. Un journaliste avec qui nous nous sommes entretenus s'était inventé un tic pour obtenir des congés et aller voir sa femme et ses enfants. Ce tic, au niveau de la main, l'empêchait de se mettre au garde à vous et de tenir une arme. Par ailleurs, l'entretien effectué avec Tevfik, docteur en philosophie, est très éclairant sur ce point. Tevfik est un appelé de 34 ans et, au vu de ses qualifications, joue de manière informelle le rôle de psychologue en plus de ses fonctions d'enseignant. Selon son avis, les gradés ont de nombreux problèmes identitaires, il mentionne le 'syndrome de Güney', c'est-à-dire les troubles psychologiques des personnes ayant combattu dans l'Est. Ecoutant et conseillant ses commandants, il était très libre de faire ce qu'il voulait. II était assez autonome et pouvait sortir de la caserne quand il le souhaitait. Pour lui, "le service militaire sert à fabriquer des menteurs, des personnes irresponsables qui se sauvent dès qu'ils peuvent de leur fonction, qui se cachent, qui utilisent les autres. Ici, il y a beaucoup de circulation et tu peux vivre en utilisant les autres, en les arnaquant'39. II explique qu'un

${ }^{39}$ Entretien réalisé le 28 juillet 2007 à Kayseri. 
Kaya, Sümbül (2009) 'La fabrique du 'soldat-citoyen' à travers la conscription en Turquie', European Journal of Turkish Studies, Thematic Issue $\mathrm{N}^{\circ} 8$, No. 8 | Surveiller, normaliser, réprimer, URL : http://www.ejts.org/document2922.html To quote a passage, use paragraph (§).

jeune s'est sauvé de la caserne et s'est promené toute la journée et ajoute : 'On n'est pas surveillé, mais plutôt on nous fait peur. Si on n'a pas peur, on fait un service comme un roi'. La séparation avec le monde civil n'est donc pas toujours si effective. De plus, une fois la période des classes terminée, les appelés ont des autorisations de sortie de fin de semaine et certains parviennent à échapper à la surveillance dont ils sont l'objet. Ils fréquentent alors des lieux proscrits (les cafés internet de Kayseri sont remplis d'appelés toutes les fins de semaine) et rencontrent des personnes 'inconnues', ce qui leur est formellement interdit. D'autres appelés exercent des fonctions leur permettant d'être souvent en interaction avec les civils : ils peuvent retarder leur retour à la caserne et prendre du temps pour eux s'ils ont terminé d'accomplir leur tâche à l'extérieur. Enfin, certains appelés n'hésitent pas à prendre congé physiquement de la caserne en se sauvant la nuit afin de se rendre dans des bars ou des discothèques.

[38] L'alcool et la drogue, tout comme les activités de loisirs qui sont formellement interdites, sont également des moyens d'échapper à l'emprise de l'institution militaire par l'évasion et par 'la prise de congé en esprit de l'institution' (Goffman, 1968: 243). L'appelé est donc physiquement présent et mentalement absent. L'institution militaire occupe souvent les appelés de base par des besognes inutiles. Les appelés n'obéissent pas toujours aux ordres et simulent l'accomplissement d'une tâche sans vraiment l'effectuer, comme par exemple faire semblant de ramasser les mégots de la cour. A la place de cela, ils vont rêvasser dans un coin, discuter avec d'autres appelés ou dormir. Lorsqu'ils sont deux à faire une garde, les appelés peuvent également se mettre d'accord pour que l'un dorme pendant que l'autre surveille. Les arrangements et compromis entre appelés peuvent aussi être un moyen souvent efficace de se soustraire solidairement aux ordres et règlements. Par ailleurs, la pratique de l'écriture d'un journal intime par les appelés s'est répandue en Turquie pour les hommes enrôlés par l'armée. Le recours à cette pratique est sans aucun doute un moyen de récréer un espace intime personnel. Ce qui est frappant dans l'analyse de ces journaux, c'est le refus de la masculinisation par l'institution. Les poésies d'amour, les images mettant en scène des fleurs, des cœurs... semblent s'opposer à la conception virilisante de l'institution.

[39] Les pratiques d'évitement permettent aux appelés de se protéger en évitant les relations avec les autres et en essayant de ne pas attirer l'attention sur eux. Ils exécutent ce qu'ils ont à faire, ni plus ni moins, et ils évitent de se mettre en avant. Le récit d'Ahmet illustre ces stratégies d'évitement, il a essayé de se faire attribuer des fonctions là où il y a le moins de gradés, 
Kaya, Sümbül (2009) 'La fabrique du 'soldat-citoyen' à travers la conscription en Turquie', European Journal of Turkish Studies, Thematic Issue $\mathrm{N}^{\circ} 8$, No. 8 | Surveiller, normaliser, réprimer, URL : http://www.ejts.org/document2922.html To quote a passage, use paragraph (§).

car il allait sans doute commettre des erreurs en les côtoyant au quotidien. II explique qu'il s'est caché à plusieurs reprises afin d'échapper à un contrôle qui allait être effectué ou lorsqu'il devait rencontrer un commandant : 'A la première occasion, on s'éclipse. Et si tu ne peux pas t'éclipser, c'est terminé'40. L'intériorisation de la hiérarchie militaire et de la position subalterne qu'occupent certains appelés, les poussent à se 'planquer', à se cacher afin d'éviter les rapports de force et de domination.

[40] Les pratiques commerciales sont normalement formellement interdites à la caserne. L'institution militaire tient à garder le monopole du commerce à l'intérieur de la caserne et bannit les échanges économiques qui lui échapperaient. Mais, dans certaines casernes qui sont loin des centres ville, il est possible d'acquérir de nombreux produits. Certains appelés terminent leur service militaire en ayant accumulé des bénéfices importants. Par exemple, ils procurent aux appelés qui sont interdits de sortie de fin de semaine des cigarettes par le biais de ce commerce illégal à un prix plus élevé que dans les commerces.

[41] Les pratiques religieuses peuvent également être considérées comme un moyen de résistance face à l'idéologie officielle de l'institution. Un imam islamiste nous a raconté qu'il avait été très tranquille pendant son service militaire et qu'il a même pu apprendre par cœur la moitié du Coran. II précise aussi que contrairement à d'autres appelés kémalistes, il n'a pas publiquement insulté l'institution. II accomplissait ses obligations et lorsqu'il avait du temps, il le consacrait à l'apprentissage du Coran. Un autre appelé, très croyant, dit avoir fait construire un lieu de prière au sein de la caserne avec de l'argent collecté auprès des membres d'une confrérie. L'armée étant en théorie le gardien du kémalisme et de la laïcité, ces pratiques montrent à quel point les individus peuvent avoir des usages non conformes de l'institution et s'opposer par là à l'esprit de l'institution. Ce qui rejoint l'idée de Goffman que 'la force de ses convictions religieuses ou politiques prémunit le vrai croyant contre les coups de l'institution totalitaire' (Goffman 1968 : 111). Dans ces exemples, le passage par l'institution confirme et renforce les convictions personnelles. Là encore, il s'agit pour certains appelés d'une protection de leur moi intime qui s'oppose à la conception de l'identité nationale que l'institution tente de leur faire intérioriser. Les pratiques politiques peuvent également s'envisager à l'intérieur de la caserne par la lecture de différents ouvrages qui seront frauduleusement introduits. Les débats politiques peuvent avoir lieu de manière très informelle au

${ }^{40}$ Entretien réalisé le 23 juillet 2007 à Kayseri. 
Kaya, Sümbül (2009) 'La fabrique du 'soldat-citoyen' à travers la conscription en Turquie', European Journal of Turkish Studies, Thematic Issue $\mathrm{N}^{\circ} 8$, No. 8 | Surveiller, normaliser, réprimer, URL : http://www.ejts.org/document2922.html To quote a passage, use paragraph $(\S)$.

sein de la caserne. Les murs des toilettes sont souvent le lieu d'expression des clivages politiques présents en Turquie. Plus l'appelé a été confronté à des institutions différentes et à des expériences antérieures diversifiées, plus il sera enclin à contester le modèle que l'institution tente de lui imposer et à refuser le rôle que lui assigne l'institution. Par exemple, si l'appelé a été confronté à des socialisations politiques qui s'opposent au kémalisme (les islamistes, les kurdistes) et plus il pourra avoir tendance à maintenir une certaine distance à son rôle. Inversement, si l'appelé possède au préalable une socialisation politique kémaliste, plus il pourra avoir tendance à se conformer à son rôle.

[42] L'institution produit donc un ethos militaire et réactive l'habitus national mais les effets sont clairement différenciés en fonction des expériences antérieures des appelés, de leurs trajectoires biographiques, et de leurs ressources.

\section{Conclusion}

[43] Le service militaire en Turquie est bien une institution totale en ce que les dispositifs disciplinaires et les formes 'd'éducation totale' présentes au sein de l'institution militaire visent à produire un ethos militaire non durable et systématique rendant possible l'incorporation par les appelés d'un habitus national conforme à l'idéologie kémaliste qui lui sera plus durable mais non homogène. En effet, la légitimité sociale de l'armée et du service militaire au sein de la population, les rites de départ et les rites d'intégration nous permettent de soutenir l'idée que le service militaire est à la fois un 'rite de passage' et un 'rite d'institution' qui affectent la prise de rôle des appelés. Les rites d'intégration (l'isolement, l'uniformisation, la 'mortification') permettent une certaine 'mise en condition' de l'appelé à son nouvel environnement dont les normes et codes diffèrent sensiblement de celles de la vie civile. Les dispositifs disciplinaires formels et informels vont procéder à la normalisation disciplinaire des appelés (la normalisation par l'examen, la normalisation par l'emploi du temps, la normalisation par la surveillance, la normalisation par le système gratification/coercition/violence). Les dispositifs disciplinaires via le processus de normalisation visent à faire intérioriser aux appelés un ethos militaire (transformations de l'hexis corporelle et du langage). Nous avons montré que l'ethos militaire n'était pas intégré de façon durable par les appelés mais la fonction de cet ethos militaire est très importante car elle vise à préparer les corps des appelés et à les rendre 'dociles et utiles'. L'assujettissement des corps va donc de pair avec la formation des 
Kaya, Sümbül (2009) 'La fabrique du 'soldat-citoyen' à travers la conscription en Turquie', European Journal of Turkish Studies, Thematic Issue $\mathrm{N}^{\circ} 8$, No. 8 | Surveiller, normaliser, réprimer, URL : http://www.ejts.org/document2922.html To quote a passage, use paragraph (§).

esprits (formation à la citoyenneté) et nous pouvons en conclure que l'ethos militaire est un outil indispensable à la réactivation de l'habitus national conforme à l'idéologie kémaliste de l'Etat Turc.

[44] Cependant, l'institution militaire ne peut produire un ethos militaire uniformément docile à l'incorporation d'un habitus national homogène. Cette homogénéisation est très partielle car les capitaux économiques, sociaux et culturels des individus vont déterminer la fonction et la place qui vont leur être attribuées par l'institution militaire. De plus, d'autres facteurs viennent influencer la tenue du rôle par l'appelé : l'âge d'incorporation, l'origine sociale, culturelle et linguistique, l'origine rurale ou urbaine, les orientations politiques et idéologiques, les expériences et les trajectoires sociales. Ces facteurs influencent également le recours par les appelés à des stratégies d'adaptation, de résistances ou de comportements déviants. La protection par les appelés de leur intimité, de leur for intérieur, à travers des tactiques conscientes ou inconscientes de résistance, fait office de barrière à l'emprise de l'armée. Ils ne correspondent pas toujours aux attentes de l'institution et réagissent de façon très variable selon les circonstances. En outre, les socialisations antérieures sont autant de ressources qui leur permettent de mieux résister au processus de normalisation de l'institution. Plus l'appelé a été confronté à des institutions différentes et à des expériences antérieures fortes, plus il sera enclin à contester le modèle que l'institution tente de lui imposer et à refuser le rôle que lui assigne l'institution. Inversement, plus l'appelé a été soumis à une socialisation 'kémaliste', plus il aura tendance à se conformer au modèle du bon 'citoyen soldat'. 
Kaya, Sümbül (2009) 'La fabrique du 'soldat-citoyen' à travers la conscription en Turquie', European Journal of Turkish Studies, Thematic Issue $\mathrm{N}^{\circ} 8$, No. 8 | Surveiller, normaliser, réprimer, URL : http://www.ejts.org/document2922.html To quote a passage, use paragraph (§).

References

Altınay, Ayşe Gül (2004) The Myth of the Military-Nation, Palgrave Macmillan, New York.

Berger, Peter; Luckmann, Thomas (2006) La construction sociale de la réalité, Paris, Armand Colin.

Bessin, Marc (2002) Autopsie du service militaire, 1965-2001, Paris, Autrement.

Bourdieu, Pierre (1976) 'Le sens pratique', Actes de la Recherche en Sciences Sociales, $\mathrm{n}^{\circ}$ 1, pp.43-86.

Bourdieu, Pierre (1978), Questions de sociologie, Paris, Les éditions de Minuit.

Bourdieu, Pierre (1980) Le sens pratique, Paris, Les éditions de Minuit.

Bourdieu, Pierre (1982) 'Les rites comme actes d'institution', Actes de la Recherche en Sciences Sociales, $n^{\circ 1}$, pp. 58-63.

Bozdemir, Mevlüt (1980) Le rôle politique de l'armée en Turquie, thèse de doctorat en science politique, Paris 1.

Caplow, Theodore; Vennesson, Pascal (2000) Sociologie militaire, Paris, Armand Colin.

Çobano lu,Özkül; Özarslan, Metin ; Yıldırım, Dursun (2004) Liseler için, Halk Bilimi, Istanbul Devlet Kitapları, Birinci Baskı, pp.132-133.

Copeaux, Etienne (1997) Espaces et temps de la nation turque. Analyse d'une historiographie nationaliste, Paris, CNRS-Editions.

Copeaux, Etienne (2000) 'Le consensus obligatoire', in Rigoni, Isabelle (dir.) Turquie : les milles visages, Paris, Editions Syllepse, pp. 89-104.

Corcuff, Philippe (1999) 'Acteur pluriel contre habitus ?', Politix, 48, 1999, pp. 157-173.

Darmon, Muriel (2007) La socialisation, Paris, Armand Colin.

Delzescaux, Sabine (2003) Nobert Elias Civilisation et décivilisation, Paris, L'Harmattan.

E itim Bro ürü (Le petit livre du soldat) (sd).

Elias, Nobert (1989) The Germans, United Kingdom, Polity Press.

Elias, Nobert (1991) La société des individus, Paris, Fayard.

Eren, Ramazan (2003) Çanakkale Kahramaları, Çanakkale, Nesil Matbaacııık.

Faguer, Jean-Pierre (1991) 'Les effets d'une 'éducation totale", Actes de la Recherche en Sciences Sociales, Volume 86, $n^{\circ} 1$, pp. 25-43.

Foucault, Michel (1975) Surveiller et punir, Paris, Gallimard.

Foucault, Michel (2001) Dits et écrits II, 1976-1988, Paris, Quarto Gallimard. 
Kaya, Sümbül (2009) 'La fabrique du 'soldat-citoyen' à travers la conscription en Turquie', European Journal of Turkish Studies, Thematic Issue $\mathrm{N}^{\circ} 8$, No. 8 | Surveiller, normaliser, réprimer, URL : http://www.ejts.org/document2922.html To quote a passage, use paragraph (§).

Foucault, Michel (2004) Sécurité, territoire, population. Cours au Collège de France (197778), Paris, Gallimard/Seuil (Collection 'Hautes Études').

Goffman, Erving (1968) Asiles: études sur la condition sociale des malades mentaux, Paris, Les éditions de Minuit.

Kaya, Sümbül (2004) 'Liberté religieuse et Répression à Kayseri', mémoire de DEA, Institut d'étude politique d'Aix-en Provence.

Lahire, Bernard (2001) L'homme pluriel, Paris, Armand Colin/Nathan.

Lahire, Bernard (2003) 'The habitus to an individual heritage of dispositions. Towards a sociology at the level of the individual', Poetics, Journal of Empirical Research on Culture, the Media and the Arts, Elsevier Science, vol. 31, septembre 2003, pp. 329-355.

Neveu, Erik (1997) 'Elias et les Allemands', Revue française de science politique, 1997, nº 5, pp. 645-653.

Noiriel, Gérard (2001) Etat, nation et immigration. Vers une histoire du pouvoir, Paris, Belin.

Pinto, Louis (1975) 'L'armée, le contingent et les classes sociales', Actes de la recherche en sciences sociales, $n^{\circ} 3$, pp. 18-41.

Ünsaldi, Levent (2005) Le militaire et la politique en Turquie, Paris, L'Harmattan.

Van Gennep, Arnold (1981), Les rites de passage, Paris, Editions A.et J.Picard.

YSE Eğitici Eğitimi, Formation aux formateurs à la Formation à l'amour de la patrie.

Yurt Sevgisi Bilinci Kazandırma Eğitimi (Projet de développement des connaissances d'amour de la patrie). 